\title{
Injective Domination in Fuzzy Graphs
}

\author{
Abdullah Q. AL-Mekhlafi \\ Department of Mathematics \\ Faculty of Education and Sciences \\ Sheba Region University, Marib, Yemen
}

\author{
Mahiuob M.Q. Shubatah \\ Department of Mathematics \\ Faculty of Sciences and Education \\ AL-Baydha University, AL-Baydha, Yemen
}

\begin{abstract}
In this paper, the concepts of injective neighbourhood and injective domination number in fuzzy graph $G$ was introduced and investigated and are denoted by $N_{i n}$ and $\gamma_{i n}$, respectively. we investigated and studied the relationship of $\gamma_{i n}$ and $N_{i n}$ with the others parameters in fuzzy graph. Finally, we collocated $N_{i n}$ and $\gamma_{i n}$ for some standard fuzzy graphs.
\end{abstract}

\section{General Terms}

AMS Mathematics Classification, (2010), 03E72, 68R10, 68R05

\section{Keywords}

Fuzzy graph, Injective neighbourhood, Injective domination number

\section{INTRODUCTION}

Graph theory is an important area of applied mathematics with a broad spectrum of applications is many fields specially in emerging fields, such as network security, computer sciences and data analysis, bio informatics, operation research, engineering and manufacturing, physics and chemistry, linguistics, or social sciences. A mathematical frame work to describe the phenomena of uncertainty in read life situation is first suggested by L.A. Zadah in (1965) [14]. Rosenfied [11] introduced the nation of fuzzy graph and several fuzzy analogs of graph theoretic concepts such as path, cycle and connectedness. The study of dominating set in graph was begun by Ore and Berge. V.R. kulli [10] work on theory of domination in graph. A. Somasundaram and D.Somasundaram [12] presented the concept of domination in fuzzy graphs. In (2011) A. Alwardi, N.D. Soner and Karam Ebadi[2] introduced and studied the concept of common neighbourhood dominating set $C N-$ domination. Perhaps the fastest growing area within graph and fuzzy graph is the study of domination, the reason being its many and varied applications in such fields as social sciences, communication networks, algorithm designs, computational complexity etc. There are several types of domination depending upon the nature of domination. Which motivated us to introduce the concepts of injective dominating set and injective domination number of fuzzy graph which denoted by $\gamma_{i n}$ using effective edges. We obtain some interesting results for this Parameter in fuzzy graphs. The relationship of $\gamma_{i n}$ with some others parameters in fuzzy graph was investigated, $\gamma_{i n}$ for some standard fuzzy graph is calculated.

\section{PRELIMINARIES}

In this section, we review some basic definitions related injective neighbourhood and injective domination of graphs, also basic definitions related to fuzzy graphs and domination in fuzzy graphs. Let $G$ be simple graph with vertex set $V(G)=\left\{v_{1}, v_{2}, \ldots, v_{n}\right\}$. For $i \neq j$, the injective neighborhood (Inj-neighborhood) of the vertices $v_{i}$ and $v_{j}$, is the set of vertices, different from $v_{i}$ and $v_{j}$, which are adjacent to both $v_{i}$ and $v_{j}$ and denoted by $\Gamma\left(v_{i}, v_{j}\right)$. For any vertex $u \in V(G)$, the open Inj - neighbourhood of $u$ denoted by $N_{i n}(u)$ is defined as $N_{i n}(u)=\{v \in V(G)$ : $|\Gamma(u, v)| \geq 1\}$. The cardinality of $N_{i n}(u)$ is called the injective neighbourhood degree (Inj - neighbourhood degree) of $u$ and denoted by $d_{i n}(u)$ in $G$, and $N_{i n}[u]=N_{i n}(u) \cup\{u\}$ is the closed (Inj - neighbourhood) of $u$. The maximum and minimum injective degree of a graph $G$ are denoted respectively by $\Delta_{i n}(G)$ and $\sin (G)$. That is $\Delta_{i n}(G)=\max _{u \in V}\left\{\left|N_{i n}(u)\right|\right\}$ and $\delta_{i n}(G)=\min _{u \in V}\left\{\left|N_{i n}(u)\right|\right\}$. If $u$ and $v$ are any two adjacent vertices in $V$ such that $|\Gamma(u, v)| \geq 1$, then we say that $u$ is injective neighbourhood (Inj-neghbourhood) of $v$ or $u$ is injective dominate (Inj-dominate) $v$. Let $G=(V, E)$ be a graph and $u \in V$ such that $|\Gamma(u, v)|=0$ for all $v \in N(u)$. Then $u$ is in every injective neighbourhood dominating set, such vertices are called injective isolated (Inj-isolated) vertices. Let $I_{i n}$ denote the set of all injective isolated vertices of $G$. Hence $I_{s} \subseteq I_{i n} \subseteq D$, where $I_{s}$ is the set of isolated vertices and $D$ is the minimum Inj-dominating set of $G$. A subset $S$ of $V$ is called an injective neighbourhood independent set (Inj-independent) set, if for every $u \in S, v \notin N_{i n}(u)$ for all $v \in S-\{u\}$. It is clear that every independent set is Inj-independent set. The Inj-independent set $S$ is called maximal if any vertex set properly containing $S$ is not $I n j-i n d e p e n d e n t$ set. The maximum fuzzy cardinality taken over all the Inj - independent set is called injective neighbourhood independence number ( Inj-independence number) and is denoted by $\beta_{i n}$ and the lower Inj-independence number $i_{i n}$ is the minimum fuzzy cardinality taken over all the Inj - maximal independent set. Let $G=(V, E)$ be a graph a subset $D$ of $V$ is called neighbourhood dominating ( $I n j-$ dominating set) if for every vertex $v \in V-D$, there exists a vertex $u \in D$ such that $u v \in E(G)$ and $|\Gamma(u, v)| \geq 1$, where $|\Gamma(u, v)|$ is the number of Inj - neighbourhood between the vertices $u$ and $v$. The injective-domination number $\gamma_{i n}$ is the minimum cardinality taken over all the injective dominating set of $G$. A complete fuzzy graph is a fuzzy graph $G=(\mu, \rho)$ such that $\rho(u, v)=\mu(u) \wedge \mu(v)$ for all $u, v \in V(G)$. Let $G=(\mu, \rho)$ be fuzzy graph with vertex 
set $V(G)=\left\{v_{1}, v_{2}, \ldots, v_{n}\right\}$. For $i \neq j$, the common neighborhood of the vertices $v_{i}$ and $v_{j}$, denoted by $\Gamma\left(v_{i}, v_{j}\right)$, is the set of vertices, different from $v_{i}$ and $v_{j}$, which are adjacent to both $v_{i}$ and $v_{j}$. A fuzzy graph $G=(\mu, \rho)$ is said to be bipartite if the vertex set $V$ can be partitioned into two nonempty sets $V_{1}$ and $V_{2}$ such that $\rho(u, v)=0$ if $u, v \in V_{1}$ or $u, v \in V_{2}$. Further, if $\rho(u, v)=\mu(u) \wedge \mu(v)$ for all $u \in V_{1}$ and $v \in V_{2}$. Then $G$ is called complete bipartite fuzzy graph and is denoted by $K_{\mu_{1}, \mu_{2}}$ where $\mu_{1}$ and $\mu_{2}$ are, respectively. Let $G=(\mu, \rho)$ be a fuzzy graph. If $0 \leq \alpha \leq t \leq 1$. Then $\left(\mu_{t}, \rho_{t}\right)$ is a subgraph of $\left(\mu_{\alpha}, \rho_{\alpha}\right)$. A path $P$ in a fuzzy graph $G=(\mu, \rho)$ is a sequence of distinct vertices $v_{0}, v_{1}, v_{2}, \ldots, v_{n}$ (except possibly $v_{0}$ and $v_{n}$ ) such that $\mu\left(v_{i}\right)>o, \rho\left(v_{i-1}, v_{i}\right)>0,0 \leq i \leq 1$. Here $n \geq 1$ is called the length of the path $P$. The consecutive pairs $\left(v_{i-1}, v_{i}\right)$ are called the edges of the path. Let $G=(\mu, \rho)$ be a fuzzy graph and let $u$ and $v$ are any two vertices in $G$ such that $\rho(u, v)=\mu(u) \wedge \mu(v)$ and $|\Gamma(u, v)|>0$. Then we say $u$ is common neighbourhood adjacent $(C N-$ adjacent $)$ to $v$ or $u$ is $C N-$ dominate $v$. Let $G=(\mu, \rho)$ be a fuzzy graph a subset $D$ of $V$ is called common neighbourhood dominating set $(C N-$ dominating $)$ if for every vertex $v \in V-D$, there exists a vertex $u \in D$, such that $\rho(u, v)=\mu(u) \wedge \mu(v)$ and $|\Gamma(u, v)|>0$, where, $\Gamma(u, v)$ is the cardinality of common neighbourhood set between the vertices $u$ and $v$. Let $G=(\mu, \rho)$ be a fuzzy graph a common neighbourhood dominating set $D$ is said to be minimal common neighbourhood dominating set if $D-\{u\}$ is not common neighbourhood dominating set of $G$ for all $v \in D$. A minimal common neighbourhood dominating set $D$ is called minimum common neighbourhood dominating set of $G$ if $|D|=\gamma_{c n}(G)$ and is denoted by $\gamma_{c n}-$ set. The common neighbourhood domination number $(C N-$ domination $)$ is the minimum fuzzy cardinality taken over all minimal common neighbourhood dominating sets of $G$ and is denoted by $\gamma_{c n}(G)$ or $\gamma_{c n}$.

\section{INJECTIVE NEIGHBOURHOOD IN FUZZY GRAPH}

Definition 1. Let $G=(\mu, \rho)$ be fuzzy graph with vertex set $V(G)=\left\{v_{1}, v_{2}, \ldots, v_{n}\right\}$. For $i \neq j$, the common neighborhood set of the vertices $v_{i}$ and $v_{j}$, is denoted by $\Gamma\left(v_{i}, v_{j}\right)$, is the set of vertices different from $v_{i}$ and $v_{j}$, which are adjacent to both $v_{i}$ and $v_{j}$.

DEFINITION 2. Let $G=(\mu, \rho)$ be a fuzzy graph for any vertex $u \in V$ the Inj - neighbourhood of $u$ denoted by $N_{i n}(u)$ is defined as $N_{i n}(u)=\{v \in V(G):|\Gamma(u, v)|>0\}$.

Definition 3. The fuzzy cardinality of $N_{i n}(u)$ is called the injective neighbourhood degree of $u$ and denoted by $\operatorname{deg}_{\text {in }}(u)=$ $\left\{\left|N_{i n}(u)\right|: v \in V(G)\right\}$. The maximum and minimum injective neighbourhood degree of a vertex in $G$ are denoted by $\Delta_{i n}(G)$ and $\delta_{i n}(G)$, respectively. That is $\Delta_{i n}(G)=\max _{u \in V}\left\{\left|N_{i n}(u)\right|\right\}$ and $\delta_{i n}(G)=\min _{u \in V}\left\{\left|N_{i n}(u)\right|\right\}$.
EXAMPLE 1. For the fuzzy graph $G$ shown in the Figure 3.1.

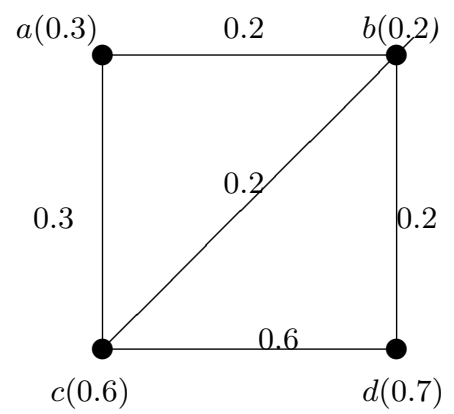

Fig 3.1.

From above Figure, we have $N_{i n}(a)=\{b, d\}, N_{\text {in }}(b)=$ $\{a, c, d\}, N_{\text {in }}(c)=\{b, d\}, N_{\text {in }}(d)=\{a, b, c\}$. Then $\operatorname{deg}_{\text {in }}(a)=$ $0.9, \operatorname{deg}_{\text {in }}(b)=1.5, \operatorname{deg}_{\text {in }}(c)=0.9$ and deg $g_{\text {in }}(d)=1.1$. Therefore

$\Delta_{\text {in }}(G)=1.5$, and $\delta_{i n}(G)=0.9$

\section{INJECTIVE DOMINATING SET IN FUZZY GRAPHS}

DEFINITION 4. Let $G=(\mu, \rho)$ be a fuzzy graph and $u, v$ are any two vertices in $G$, if $u \in N_{i n}(v):|\Gamma(u, v)|>0$, we say that $v$ is $i n j-$ dominates $u$.

Definition 5. Let $G=(\mu, \rho)$ be a fuzzy graph, a vertex subset $D$ of $V$ is called injective dominating set, (in short, Inj-dominating), if for every vertex $v \in V$ either $v \in D$ or there exists a vertex $u \in D$, such that $|\Gamma(u, v)|>0$.

Definition 6. Let $G=(\mu, \rho)$ be a fuzzy graph, an injective dominating set $D$ is said to be minimal injective dominating set if $D-\{u\}$ is not injective dominating set of $G$ for all $u \in D$.

DEFINITION 7. The minimum fuzzy cardinality taken over all minimal injective dominating sets in fuzzy graph $G$ is called the injective domination number of $G$ and is denoted by $\gamma_{i n}(G)$ or simple $\gamma_{i n}$

Definition 8. Let $G=(V, E)$ be a fuzzy graph, a subset $S$ of $V$ is called injective independent set $I n j-$ independent set if for every $u \in S, v \notin N_{i n}(u)$ for all $v \in S-\{u\}$.

DeFINITION 9. Let $G=(\mu, \rho)$ be a fuzzy graph, an injective independent set $S$ is said to be maximail injective independent set if $S-\{u\}$ is not injective independent set of $G$, for all $u \in S$.

DEFINITION 10. The maximal fuzzy cardinality taken over all maximail injective independent sets in fuzzy graph $G$ is called the injective independence number of $G$ and is denoted $\beta_{i n}(G)$.

In the following Example, we explain the concept of injective dominating set.

ExAmple 2. Consider the fuzzy graph $G$ given in the Figure (3.1). $D_{i n 1}=\{b\}, D_{i n 2}=\{a, c\}$ and $D_{i n 3}=\{d\}$ are minimal injective dominating sets. Then $\left|D_{1 i n}\right|=|\{b\}|=0.2=\gamma_{i n}(G)$. 
DEFINITION 11. Let $G=(\mu, \rho)$ be a fuzzy graph the injective complement of $G$ is denoted by $\overline{G_{i n}}$ such that $V(G)=V\left(\overline{G_{i n}}\right)$ and any two vertices $u$ and $v$ in $\overline{G_{i n}}$ are adjacent if and only if they are not $I n j-$ adjacent in $G$.

THEOREM 12. Let $G=(\mu, \rho)$ be a fuzzy graph if $G=P_{n}$ or $G=C_{n}$ path or cycle with $n$ vertices $n \geq 3$

$$
\gamma_{i n}(G) \leq \frac{p}{2}
$$

PROOF. Let $G$ be a fuzzy graph path or cycle with $n$ vertices, such that $n \geq 3$ and let $D$ be an injective dominating set of $G$. Then every vertex $v \in V-D$ there exists a vertex $u \in D$, such that $\mid \Gamma(u, v)>0$. Also the distance between $u$ and $v$ is at least equal to the wight of two edge, i.e $u$ and $v$ are Inj-vertices. Hence,

$$
\gamma_{i n}(G) \leq \frac{p}{2}
$$

THEOREM 13. Let $G$ be any fuzzy graph. Then the injective dominating set $D_{\text {in }}$ of $G$, is minimal injective dominating set if and only if one of the following conditions holds:

(i) $N_{i n}(u) \cap D_{i n}=\phi$;

(ii) There is a vertex $v \in V-D_{i n}$, such that $N_{i n}(v) \cap D_{i n}=\{u\}$.

Proof. Let $G$ be a fuzzy graph and let $D_{\text {in }}$ be a minimal injective dominating set of $G$. Then $D_{i n}-\{v\}$ is not injective dominating set and there exists a vertex $u \in V-D_{i n}-\{v\}$ such that $u$ is not injective dominated by any vertex of $D_{i n}-\{v\}$ if $u=v$, then $N(u) \cap D_{\text {in }}=\phi$, if $u \neq v$. Then $N(v) \cap D_{\text {in }}=\{u\}$.

Conversely, Suppose that $D_{\text {in }}$ is injective dominating set and for each vertex $u$ in $D_{\text {in }}$ one of the two condition holds.

Now, we want to prove that $D_{\text {in }}$ is minimal. Suppose $D_{\text {in }}$ is not minimal. Then there exists a vertex $v \in D_{\text {in }}$ such that $D_{i n}-\{v\}$ is injective dominating set. Thus, $u$ is an Inj - adjacent nieghbour to at least one vertex in $D_{i n}-\{v\}$. Hence, condition $(i)$ does not hold, also if $D_{i n}-\{v\}$ is injective dominating set. Then every vertex in $V-D_{\text {in }}$ is an Inj-adjacent to at least one vertex in $D_{i n}-\{v\}$. That means condition (ii) does not hold. So we get contradiction. Hence, $D_{i n}$ is minimal injective dominating set.

DEFINITION 14. A vertex $u$ in a fuzzy graph $G$, is called an injective isolated vertex in $G$ if $|\Gamma(u, v)|=0, \forall v \in V(G)$.

THEOREM 15. Let $G$ be a fuzzy graph without injectiveisolated vertices if $D_{i n}$ is minimal injective dominating set. Than $V-D_{i n}$ is an injective dominating set.

Proof. Let $D_{i n}$ be minimal injective dominating set of $G$. Suppose that $V-D_{\text {in }}$ is not injective dominating set. Then there exists a vertex $u$ in $D_{i n}$ such that $u$ is not injective dominated by any vertex in $V-D_{i n}$. Then $u$ is injective dominated by at least one vertex $v$ in $D_{\text {in }}-\{u\}$. Thus, $D_{\text {in }}-\{u\}$ is an injective dominating set of $G$ which contradicts to the minimiality of the injective dominating set $D_{i n}$. Therefore, every vertex in $D_{i n}$ has common neighbourhood with at least one vertex in $V-\left\{D_{\text {in }}\right\}$. Hence, $V-\left\{D_{i n}\right\}$ is an injective dominating set of $G$.

If $u$ is an injective-isolated vertex. Then $u$ is in every injective dominating set.

THEOREM 16. For any fuzzy graph $G$.

$$
\gamma_{i n}(G) \leq \gamma_{c n}(G)
$$

Proof. Since every $C N-$ dominating set of fuzzy graph is an injective dominating set of fuzzy graph $G$. Hence

$$
\gamma_{i n}(G) \leq \gamma_{c n}(G)
$$

THEOREM 17. Let $G=(\mu, \rho)$ be a fuzzy graph and $S$ is maximal Inj-independent set. Then $S$ maximal injective dominating set.

Proof. Let $S$ maximal Inj - independent set and $u \in$ $V-S$ if $u \notin N_{\text {in }}(v)$ for every $v \in S$ then $S \bigcup\{u\}$ is an Inj - independent set contradiction. Therefor $u \in N_{i n}(v)$ for some $v \in S$. Hence $S$ injective dominating set. We went prove that $S$ is maximal injective dominating set. Than there exist some vertex in $S$ has a common neighbors with $u$. Which is contradiction. Therefore, $S$ is maximal injective dominating set.

THEOREM 18. For any complete fuzzy graph $G=K_{\mu}$. Then

$$
\gamma_{i n}\left(K_{\mu}\right)=\min \left\{\mu(v): v \in V\left(K_{\mu}\right)\right\} .
$$

Proof. Let $u$ and $v$ be any two vertices in $K_{\mu}$. Then $|\Gamma(u, v)|>0$ and every vertex of $G$ form an injective dominating set of $G$ Hence

$$
\gamma_{i n}\left(K_{\mu}\right)=\min \left\{\mu(v): v \in V\left(K_{\mu}\right)\right\} .
$$

THEOREM 19. For any complete bipartite fuzzy graph $G=$ $K_{r, m}$, with $\left|V_{1}\right|=r$ and $\left|V_{2}\right|=m$. Than

$$
\gamma_{i n}\left(K_{r, m}\right)=\min \left\{\mu\left(u_{i}\right) ; u_{i} \in V_{1}\right\}+\min \left\{\mu\left(v_{j}\right) ; v_{j} \in V_{2}\right\} .
$$

Proof. Let $G$ be complete bipartite fuzzy graph. Then $\rho(u, v)=0$ and $\Gamma(u, v)=$ ofor all $u, v \in V_{1}$ or $V_{2}$ and $\rho(u, v)=$ $\mu(u) \wedge \mu(v), \forall u \in V_{1}$ and $v \in V_{2}$. Thus every vertex in $V_{1}$ has an Inj - neighbourhood with every vertex in $V_{1}$. and every vertex of $V_{2}$ has an Inj - neighbourhood with every vertex in $V_{2}$. Then $D_{\text {in }}$ contains two vertices one of $V_{1}$ and the other from $V_{2}$. Hence, $\gamma_{i n}\left(K_{r, m}\right)=\min \left\{\mu\left(u_{i}\right) ; u_{i} \in V_{1}\right\}+\min \left\{\mu\left(v_{j}\right) ; v_{j} \in V_{2}\right\}$.

For any star fuzzy graph $G=K_{t, P-t}, t=\mu(u)$, $u$ is a root

$\left.\gamma_{i n}\left(K_{t, P-t}\right)=\mu(u)+\min \left\{\mu\left(v_{i}\right) ; \forall v_{i} \in V-\{u\}\right)\right\}$, u isarootvertex

Proof. Let $G=K_{t, P-t}$ be a star fuzzy graph. By the above Theorem, $V_{1}=\{u\}$ and $V_{2}=\left\{v_{1}, \ldots . ., v_{m}\right\}$. Then $D_{\text {in }}$ contains root vertex and one vertex of $V_{2}$. Hence, $\gamma_{i n}\left(K_{t, P-t}\right)=\mu(u)+$ $\left.\min \left\{\mu\left(v_{i}\right) ; v_{i} \in V-\{u\}\right)\right\}$.

THEOREM 20. For any wheel fuzzy graph with $p$ vertices.

$$
\gamma_{i n}\left(W_{p}\right)=\min \left\{\mu(v): v \in V\left(W_{p}\right)\right\} .
$$

PROOF. Since every vertex in $W_{p}$ has Inj - neighborhood. Than every vertex form an injective dominating set of $W_{p}$. Hence

$$
\gamma_{i n}\left(W_{p}\right)=\min \left\{\mu(v): v \in V\left(W_{p}\right)\right\} .
$$

THEOREM 21. For any fuzzy graph $G=(\mu, \rho)$. Then

$$
\gamma_{i n}(G) \leq p-\Delta_{i n}(G) .
$$


Proof. Let $G=(\mu, \rho)$ be any fuzzy graph and let $v \in V(G)$, such that $d_{i n}(v)=\Delta_{i n}(G)$, for each vertex $v \in N_{i n}(u), v$ has a common neighbor with $u$. Thus $V-N_{i n}(v)$ is an injective dominating set. Therefore,

$\gamma_{i n} \leq\left|V-N_{i n}(v)\right|=p-\Delta_{i n}(G)$.

For any fuzzy graph $G=(\mu, \rho)$. Then

$$
\gamma_{i n}(G) \leq p-\delta_{i n}(G) .
$$

Proof. Since $\delta_{i n} \leq \Delta_{i n}$ and by the above Theorem.. Then $\gamma_{i n}(G) \leq p-\delta_{i n}(G)$.

THEOREM 22. For any fuzzy graph $G=(\mu, \rho)$. Then

$$
\gamma_{i n}(G) \leq \beta_{i n}(G) .
$$

Proof. Let $G$ be a fuzzy graph, and $S$ is maximail Inj independent set of $G$, such that $|S|=\beta_{\text {in }}(G)$. Then every vertex $v \in V-S$ is an Inj-adjacent to at least one vertex of $S$. Thus $S$ is an injective dominating set. Hence,

$$
\gamma_{i n}(G) \leq \beta_{i n}(G) \text {. }
$$

THEOREM 23. Every injective dominating set in a fuzzy graph $G=(\mu, \rho)$ is injective dominating set in crisp graph $G^{*}=(V, E)$, but the converse is not true.

Proof. Let $G=(\mu, \rho)$ be a fuzzy graph, with injective dominating set $D_{\text {in }}$ and let $u \in D_{i n}$. Then there exists $v \in N_{i n}(u)$ such that $|\Gamma(u, v)|>0$

Since every Inj-neighbourhood set in fuzzy graph $G$ is an Injneighbourhood set in crisp graph $G^{*}$, so $v \in N_{\text {in }}(u),|\Gamma(u, v)| \geq$ 1 and $u$ is dominates $v$ in $G^{*}$. Thus $u \in D_{\text {in }}$ in $G^{*}$. Hence, $D_{\text {in }}$ is an injective dominating set in $G^{*}$.

In the following Example, we show that the converse of the above Theorem, is not true.

EXAMPLE 3. For the fuzzy graph G shown in Figure 3.2.

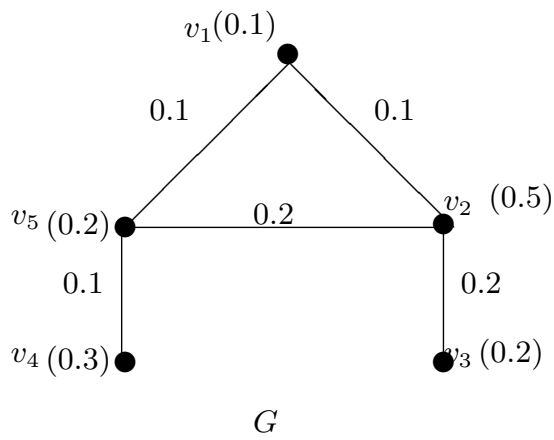

Fig. 3.2

From the Figure 3.2, $D_{i n}=\left\{v_{1}\right\}$, is minimal injective dominating set of $G^{*}$ with minimum cardinality, but $D_{\text {in }}$ is not injective dominating set of $G$. Also $S_{i n}=\left\{v_{1}, v_{4}\right\}$ is minimal injective dominating set of $G$. and $S$ is an injective dominating set of $G^{*}$.

THEOREM 24. Let $G=(\mu, \rho)$ be a fuzzy subgraph of $G^{*}=$ $(V, E)$ graph, with injective dominating set of $\left(G^{*}\right)$.

Then $\gamma_{i n}(G) \leq \gamma_{i n}\left(G^{*}\right)$. Furthermore, equality holds, if $\mu(v)=$ $1, \forall v \in V(G)$.
PROOF. By Theorem (23), every injective dominating set in fuzzy graph $G$ is injective dominating set in crisp graph $G^{*}$. Then $\gamma_{i n}(G) \leq \gamma_{i n}\left(G^{*}\right)$. and if $\mu(v)=1, \forall v \in V(G)$ we have $\gamma_{i n}(G)=\gamma_{i n}\left(G^{*}\right)$.

THEOREM 25. For any fuzzy graph $G=(\mu, \rho)$. Then

$$
\gamma_{i n}(G)+\gamma_{i n}(\bar{G}) \leq 2 p \text {. }
$$

Proof. Since $\gamma_{i n}(G) \leq p$ and $\gamma_{i n}(\bar{G}) \leq p$. Then

$$
\gamma_{i n}(G)+\gamma_{i n}(\bar{G}) \leq 2 p \text {. }
$$

Definition 26. Let $G=(\mu, \rho)$ be any fuzzy graph on a vertex set $V$ and $D_{i}, \quad i=1,2 \ldots \ldots, n$ be an injective dominating sets of $G$.Than $D=\left\{D_{1}, D_{2}, \ldots, D_{n}\right\}$ such that $\|D\|=$ $\max \left\{\sum_{i=1}^{n} \frac{\mu\left(D_{i}\right)}{n}, \quad i=1,2, \ldots . n\right\}$ is called the $\operatorname{Inj}-$ domatic of a fuzzy graph $G$ and is dented by $d_{i n}(G)$ 3.3 .

EXAMPLE 4. Consider the fuzzy graph $G$ shown in the Figure

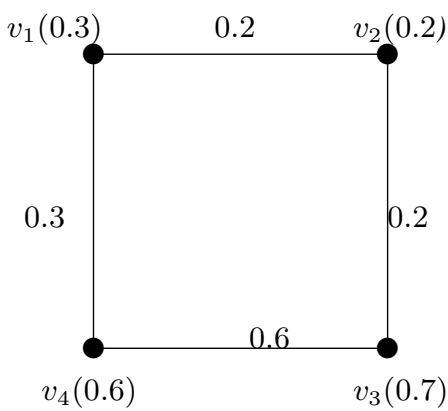

Fig 3.3.

From a Figuer (3), we have

$P_{1}=\left\{\left(v_{1}, v_{2}\right),\left(v_{1}, v_{4}\right),\left(v_{2}, v_{3}\right),\left(v_{3}, v_{4}\right)\right\}$

$P_{2}=\left\{\left(v_{1}, v_{2}, v_{3}\right),\left(v_{1}, v_{2}, v_{4}\right),\left(v_{2}, v_{3}, v_{4}\right)\right\}$

$P_{3}=\left\{v_{1}, v_{2}, v_{3}, v_{4}\right\}$

$\left\|P_{1}\right\|=\frac{0.3+0.2}{2}+\frac{0.3+0.6}{2}+\frac{0.2+0.7}{2}+\frac{0.7+0.6}{2}=1.8$

$\left\|P_{2}\right\|=\frac{0.3+0.2+0.7}{3}+\frac{0.3+0.2+0.6}{3}+\frac{0.2+0.7+0.6}{3}=1.4$

$\left\|p_{3}\right\|=\frac{0.3+0.2+0.7+0.6}{4}=0.45$

Hence $d_{i n}(G)=\max \left\{\sum_{i=1}^{n} \frac{\mu\left(D_{i}\right)}{n}, i=1,2 \ldots 8\right\}=1.8$

Theorem 27. For any fuzzy graph $G=(\mu, \rho)$. Then

$$
d_{i n}(G) \leq \delta_{i n}(G)+t .
$$

$t=\max \{\mu(v): v \in V(G)\}$

Proof. Let $G$ be any fuzzy graph, such that $d_{i n}(G)>$ $\delta_{i n}(G)+t$. Then there exist at least $\delta_{i n}(G)+t+\bar{t}$, such that $\bar{t} \in V(G)$ be injective dominating set of $G$. Let $v$ be any vertex in $V(G)$ such that $\operatorname{deg}_{i n}(v)=\delta_{i n}(G)$ Then there is at least one of the injective dominating set which has an intersection with $N_{i n}[v]$ .Hence the injective dominating set can not dominate $v$ which contradiction to our assumption. Then

$$
d_{i n}(G) \leq \delta_{i n}(G)+t .
$$


THEOREM 28. For any fuzzy graph $G=(\mu, \rho)$. Then

$$
d_{i n}(G)+\gamma_{i n}(G) \leq p+t
$$

$t=\max \{\mu(v): v \in V(G)\}$

Proof. Let $G$ be any fuzzy graph by Theorem $4.25, \gamma_{i n}(G) \leq$ $p-\Delta_{i n}(G)$. and $\gamma_{i n}(G) \leq p-\delta_{i n}(G)$ and by Theorem 4.34, $d_{\text {in }}(G) \leq \delta_{\text {in }}(G)+t$. Then

$$
\left.d_{i n}(G)+\gamma_{i n}(G)\right\} \leq p+t .
$$

THEOREM 29. For any fuzzy graph $G=(\mu, \rho)$ Then

$$
d_{i n}(G)+d_{i n}\left(\overline{G^{i n}}\right) \leq p+t .
$$

$t=\max \{\mu(v): v \in V(G)\}$.

Proof. It is clear that $d_{i n}(G)+d_{i n}\left(\overline{G^{i n}}\right) \leq \delta_{i n}(G)+t+$ $\delta_{i n} \bar{G}+t$. Also $\delta_{i n}\left(\left(\overline{G^{i n}}\right)\right)=p-t-\Delta_{i n}$. Hence

$$
d_{i n}(G)+d_{i n}\left(\overline{G^{i n}}\right) \leq p+t .
$$

\section{REFERENCES}

[1] Abdullah Q. AL-Mekhlafi, Saqr H. AL-Emrany and Mahiuob M.Q. Shubatah, Common neighbourhood and common neighbourhood domination in fuzzy graphs, Asian journal of Advanced Research and Reports, 15(1), 2020, 1-9.

[2] Alwardi A; N.D. Soner and KaramM Ebadi, On the common neighbourhood domination number; J. Comp. Math. Sci. 2(3), (2011), 547-556.

[3] Alwardi A; N.D. Soner, CN-edge domination in graphs, Vladikavkaz. Mat. Zh., 15(2), (2013), 11?17.

[4] Alwardi A; R. Rangarajan and Akram Alqesmah, On the injective domination of fuzzy graphs, Palestine Journal of Mathematics; 7(1), (2018), 202-210.

[5] Alqesmalh1 A; A. Alwardi2 and R. Rangaran3, Edge injective domination of graph, Gulf Journal of Mathematics; 5(2), (2017), 46-55.

[6] Hameh A; Ali Irnmanesh and (et al), On common neighborhood graphs, Iranian J. Math. Chem. 9 (1), (2018), 37-46.

[7] Dundar P; A. Aytac and E. Kilic; Common-neighbourhood of a graph; Bol. Soc. Paran. Mat. 35(1), (2017), 23 ?32.

[8] Haray F. Graph theory, Addison Wesley, Third printing, October, (1972).

[9] Kaufman A. Introduction a La Theorie des Sous- emsembles Flous, Paris, Masson etcie Editeurs, (1973).

[10] Kulli V.R. and L.S.C. Sigarkanti, Inverse domination in graphs, Nat. Acad. Sci. Ltt. 14, (1991), 473-475.

[11] Rosenfeld A. Fuzzy graphs, In. L.A. Zadeh, K.S.Fu and M. Shimura, (Eds). Fuzzy sets and their applications, Academic Press, (1975), 77-95.

[12] Somasundaram A. and S. Somasundaram, Domination in fuzzy graphs-I, Pattern Recognition Letters, 19, (1998), 787791.

[13] Somasundaram A. Domination in fuzzy graphs-II, Journal of Fuzzy Mathematics, 20, (2000), 281-289.

[14] Zadeh. L.A., Fuzzy Sets, Information and control, 8, 1965, 338-353. 